\title{
Sustained Improvements in EQ-5D Utility Scores and Self-rated Health Status in Patients With Ankylosing Spondylitis After Spa Treatment Including Low-dose Radon
}

Antje van der Zee-Neuen ( $\square$ antje.zee@pmu.ac.at )

Paracelsus Medical University

\section{Victoria Strobl}

Paracelsus Medical University

Heidemarie Dobias

Paracelsus Medical University

Julia Fuchs

Paracelsus Medical University

Johannes Untner

Gastein Healing Gallery

Wolfgang Foisner

Cure and Rehabilitation Center

Martina Knapp

Stiftung Kurtherme Badehospiz

Sebastian Edtinger

Kardinal Schwarzenberg Klinikum

Martin Offenbächer

Gastein Healing Gallery

Markus Ritter

Paracelsus Medical University

Bertram Hölzl

Landesklinik St. Veit im Pongau

Martin Gaisberger

Paracelsus Medical University

\section{Research Article}

Keywords:

Posted Date: February 3rd, 2022 
DOI: https://doi.org/10.21203/rs.3.rs-1306535/v1

License: (c) (1) This work is licensed under a Creative Commons Attribution 4.0 International License. Read Full License 


\section{Abstract}

\section{Background}

Patients with ankylosing spondylitis (AS) have significantly lower quality of life (QoL) than the general population. Holistic interventions addressing QoL include spa- or balneotherapy. Inclusion of radon in spa-therapy treatments is beneficial in reducing pain and shows promising results in improving QoL in AS-patients. We aimed to explore the association of spa therapy including low-dose radon with systematically monitored QoL in AS-patients over an extended timeperiod.

\section{Methods}

Registry data collected for the "Radon indication registry for the assessment of pain reduction, increase of quality of life and improvement in body functionality throughout low-dose radon hyperthermia therapy' in the Austrian Gastein valley comprising data on QoL (EuroQol EQ-5D) directly before the treatment (baseline), directly, 3; 6 and 9 months after the treatment, age, sex and body mass index (BMI) were analysed. Two linear regression models explored the association between time of measurement with 1) EQ-5D utilities and 2) EuroQol visual analogue scale (VAS) score. Alterations of 0.05 (utilities) and 5.00 (VAS) were considered clinically relevant.

\section{Results}

Two-hundred-ninety-one AS-patients were included in the analyses. Forty-four percent $(n=128)$ were women, the mean age was 52 (SD 10) and the average BMI was 26 (SD 4). Utilities and VAS were significantly higher at all timepoints compared to baseline. Improvements were clinically relevant at all timepoints in case of the EQ-5D VAS and until 6 months after treatment for the utility index.

\section{Conclusion}

Spa therapy including radon results in significant and clinically relevant improvements in Qol of ASpatients until 6-9 months after treatment.

\section{Introduction}

Ankylosing spondylitis (AS) is the most common form of the rheumatic disease group of spondyloarthritides. It occurs in approximately 23.8 per 10,000 Europeans and is more prevalent in men than women. AS affects the axial skeleton leading to inflammatory back pain, damage to physical structures as well as impairments in physical functioning. These impairments may result in reduced participation and decreased quality of life (QoL). (1-4) The growing understanding of QoL as key factor when measuring the effectiveness of healthcare interventions as well as the embracement of biopsychosocial models rather than just biological models for the evaluation of health emphasize the relevance of interventions focussing on the improvement of QoL. $(5,6)$ 
Previous research has pointed out that AS-patients have significantly lower QoL than the general population but that pharmacological treatment is beneficial in improving their QoL. Particularly the combination of anti-TNF-a therapy in combination with physical exercise may reduce the adverse effect of AS on QoL. $(7,8)$

However, the evidence on the effectiveness of alternative or complementary non-pharmacological interventions in improving QoL in AS patients is still limited. Common symptom-oriented interventions like physiotherapeutic treatment are effective in the reduction of disease activity and pain as well as the improvement of functional capacity. (9) Yet, holistic interventions have the potential of addressing a wider range of the AS-patient's health state including mental health and participation in daily life. $(10,11)$ Those aspects are particularly relevant when assessing QoL from a patient's perspective.

Holistic interventions for AS regularly comprise multidisciplinary treatments including spa- or balneotherapy/speleotherapy. Still, little is known about the effect of these interventions on QoL. Kamioka et al. summarized the body of knowledge in an overview of systematic reviews with metaanalysis based on randomized controlled trials of balneotherapy and spa-therapy from 2000 to 2019 and did not identify any review focussing on spa-therapy in relation to QoL. (12) A limited number of studies specifically addressed the effectiveness of combined spa-exercise therapy on QoL. For example, Colina et al. demonstrated that in AS patients, combining pharmacological treatment with spa-therapy resulted in significantly better QoL than pharmacological treatment alone six months after treatment initiation. (13) A randomized controlled trial by van Tubergen et al. showed that QoL, expressed by EuroQol-5D utilities, was significantly higher in patients that received spa-therapy (one with and one without radon treatment) compared to those who received usual care until 40 weeks after the treatment. In this study, the application of utilities enabled valuation of QoL from a societal perspective (i.e., utility values accounted for preferences the society has for a particular health state).(14)

Among spa-therapies, treatment with low-dose radon has shown to be effective in achieving long-term pain reduction in persons with musculoskeletal diseases (including AS) $(11,15-19)$ and showed promising results with regard to improvements in functionality $(20,21)$ as well as in QoL. $(22,23)$. However, to the best of our knowledge, until now no data exist on the association of spa therapy including radon with systematically monitored QoL in patients with AS over an extended period of time while accounting for both, QoL from a societal perspective and individually perceived QoL.

Therefore, the aim of the current study was to explore whether spa treatment including low-dose radon results in sustained significant and clinically relevant improvement of QoL in patients with AS.

\section{Methods}

The current study concerns a longitudinal analysis of registry data collected for the "Radon indication registry for the assessment of pain reduction, increase of quality of life and improvement in body functionality throughout low-dose radon hyperthermia therapy" (registration

ID ISRCTN67336967; https://doi.org/10.1186/ISRCTN67336967) in the valley of Gastein in Austria. The 
registry collects data from individuals visiting the valley of Gastein for the purpose of spa-treatment including radon for a variety of rheumatic diseases. Data are collected by means of standardized questionnaires that are completed by participants directly before commencement of the treatment (baseline), directly after the treatment and 3; 6 and 9 months after the treatment.

\section{Population}

For the current study, data provided by participants with AS were included if they completed the questionnaire at each timepoint.

\section{Intervention}

The intervention consisted of an individualized spa-treatment including radon. This so-called low-dose radon balneo/speleo therapy (LDRnBST; radon-therapy) is part of a holistic treatment program for patients with AS and is applied in terms of balneo- and/or speleotherapy. The former includes bathing in water with low activity of radon as applied by the local facilities according to standardized treatment regimens. An intervention including radon-therapy consists of approximately 10 baths with a duration of 20 minutes. Speleotherapy including radon describes the process of relaxation while being exposed to low activity of radon, high humidity and mild hyperthermia $\left(37-41.5^{\circ} \mathrm{C}\right.$ ) in the healing gallery of Gastein (a former gold mine) located in moderate altitude $(1280 \mathrm{~m})$ above sea level for an average time of 60 minutes on alternate days.

The intervention had an average duration of 17.5 days (SD 3.5) and took place in the valley of Gastein in the Austrian Alps.

\section{Outcomes}

The EuroQol EQ-5D (C EuroQol Research Foundation. EQ-5D ${ }^{T M}$ ) is a self-reported questionnaire consisting of two parts, a descriptive system comprising 5 dimensions of health (i.e., mobility, self-care, usual activities, pain/discomfort, anxiety/depression) and a visual analogue scale (VAS) capturing participant's self-rated health status on a 0-100 scale with higher values representing better health. Using the unique score from each of the 5 dimensions of health a utility index score can be calculated (i.e., von NeumannMorgenstern utility value for current health). (24)

Single values for each of the 5 dimensions reflect the level of problem with each dimension resulting in an individual health state. This health state can be converted into a weighted health state by applying scores from the EQ-5D preference weights extracted from the general population which can take a value from 0 (death) to 1 (full health).

The EQ-5D utility index and EuroQol VAS were used as outcome variables for the current study. In absence of Austrian population weights, German population weights were used to calculate the EQ-5D utility index. (25) 


\section{Main independent variable of interest and covariates}

The timepoint of survey completion by the participants was used as main independent variable of interest. Covariates were chosen a priori and included age (in years), sex (men/women) and body mass index (BMl; BMl=weight $[\mathrm{kg}] /$ height $[\mathrm{m}]^{2}$ ) due to their already established influence on health and health related QoL. (26-28)

\section{Statistical analyses}

First, descriptive statistics were used to characterize the sample in terms of age, gender and BMI at baseline (i.e., directly before the intervention) and to describe the EQ-5D utility index and VAS-score for each of the timepoints of measurement. Next, two linear regression models were computed to explore the association of timepoint of measurement with a) the EQ-5D utility index and b) the EuroQol VAS-score while adjusting for age, sex and BMI. After each model, margins and their $95 \%$ confidence interval (Cl) were calculated to produce specific age, gender and BMI standardized estimates for the utility index and VAS score.

P-values $\leq 0.05$ were considered statistically significant. A change of 0.05 in the EQ-5D utility index and of 5.00 in the EuroQol VAS was considered clinically relevant. $(29,30)$

\section{Results}

The final sample included in the analyses consisted of 291 participants who provided complete data for all timepoints. The sample consisted of 128 women, the mean age was 52 years and the average BMI was 26. Table 1 shows the unstandardized EQ-5D utility index and VAS scores for each timepoint. Figure 1 illustrates the course of the dimensions (i.e. mobility, self-care, usual activities, pain/discomfort, anxiety/depression) based on which the utility index was calculated. 
Table 1

Characteristics of study population at baseline and directly, 3, 6 \& 9 months after spa-treatment including radon

\begin{tabular}{|c|c|c|c|c|c|}
\hline & Baseline $^{a}$ & $\begin{array}{l}\text { Timepoint } \\
1^{b}\end{array}$ & $\begin{array}{l}\text { Timepoint } \\
2^{c}\end{array}$ & $\begin{array}{l}\text { Timepoint } \\
3^{d}\end{array}$ & $\begin{array}{l}\text { Timepoint } \\
4^{\mathrm{e}}\end{array}$ \\
\hline \multirow[t]{2}{*}{ Age, mean (SD); range } & $\begin{array}{l}51.80 \\
(10.14) ;\end{array}$ & & & & \\
\hline & $\begin{array}{l}19.00- \\
79.00\end{array}$ & & & & \\
\hline Women, n (\%) & $\begin{array}{l}128 \\
(44.00)\end{array}$ & & & & \\
\hline \multirow[t]{2}{*}{$\begin{array}{l}\text { Body mass index }{ }^{1} \text {, mean }(S D) \text {; } \\
\text { range }\end{array}$} & $\begin{array}{l}26.37 \\
(4.25) ;\end{array}$ & & & & \\
\hline & $\begin{array}{l}18.37- \\
39.56\end{array}$ & & & & \\
\hline \multirow[t]{2}{*}{$\begin{array}{l}\text { EQ-5D utility index }{ }^{2} \text {, mean } \\
\text { (SD); range }\end{array}$} & $\begin{array}{l}0.79 \\
(0.17)\end{array}$ & $\begin{array}{l}0.88 \\
(0.11)\end{array}$ & $\begin{array}{l}0.87 \\
(0.12)\end{array}$ & $\begin{array}{l}0.85 \\
(0.13)\end{array}$ & $\begin{array}{l}0.83 \\
(0.16)\end{array}$ \\
\hline & $0.01-1.00$ & $0.36-1.00$ & $0.24-1.00$ & $0.19-1.00$ & $0.08-1.00$ \\
\hline \multirow[t]{2}{*}{$\mathrm{EQ} \mathrm{VAS}{ }^{3}$, mean $(\mathrm{SD})$; range } & $\begin{array}{l}62.51 \\
(18.01)\end{array}$ & $\begin{array}{l}74.88 \\
(17.10) ;\end{array}$ & $\begin{array}{l}74.76 \\
(15.41)\end{array}$ & $\begin{array}{l}71.46 \\
(15.52)\end{array}$ & $\begin{array}{l}67.23 \\
(18.00)\end{array}$ \\
\hline & $\begin{array}{l}15.00- \\
100.00\end{array}$ & $\begin{array}{l}2.00- \\
100.00\end{array}$ & $\begin{array}{l}20.00- \\
100.00\end{array}$ & $\begin{array}{l}18.00- \\
98.00\end{array}$ & $\begin{array}{l}20.00- \\
100.00\end{array}$ \\
\hline \multicolumn{6}{|c|}{$\begin{array}{l}{ }^{a} \text { directly bevor intervention; }{ }^{b} \text { directly after intervention, }{ }^{c} 3 \text { months after intervention; }{ }^{d} 6 \text { months after } \\
\text { intervention; }{ }^{e} 9 \text { months after intervention }\end{array}$} \\
\hline \multicolumn{6}{|c|}{$\begin{array}{l}{ }^{1} \text { based on classification of World Health Organization: underweight } B M \mid<18.5 \text {, normal } B M \mid>=18.5 \& \\
<=24.99 \text {, overweight }>=25 \&<30 \text {, obese }>=30\end{array}$} \\
\hline \multicolumn{6}{|c|}{$\begin{array}{l}{ }^{2} \text { von Neumann-Morgenstern utility value for current health based on } 5 \text { dimensions of EuroQol (i.e. } \\
\text { mobility, self-care, usual activities, pain/discomfort, and anxiety/depression), range } 0-1 \text { (i.e. } 0=\text { death, } \\
1=\text { perfect health) }\end{array}$} \\
\hline \multicolumn{6}{|c|}{$\begin{array}{l}{ }^{3} \text { self-rated health status on a graduated }(0-100) \text { scale, } 0=\text { 'The worst health you can imagine', } 100= \\
\text { 'The best health you can imagine' }\end{array}$} \\
\hline
\end{tabular}

\section{Health over time EQ-5D utility index}

The age, sex and BMI standardized association of timepoint of measurement and utility index showed that at each timepoint, the index value was significantly higher than the baseline value indicating better health. Directly, 3 months and 6 months after the intervention the index value increased by $0.09[95 \% \mathrm{Cl}$ $0.07 ; 0.11], 0.08$ [95\% $\mathrm{Cl} 0.06 ; 0.10]$ and 0.06 [95\% $\mathrm{Cl} 0.05 ; 0.09]$, respectively. These increases reflected a 
clinically relevant change. Nine months after the intervention the utility index was still increased $(0.04$ $[95 \% \mathrm{Cl} 0.02 ; 0.06])$ but this improvement was not clinically relevant. (Table 2 )

\section{Health over time EuroQol VAS}

The age, sex and BMI standardized association of timepoint of measurement and EuroQol VAS showed that at each timepoint, the VAS score was significantly higher than the baseline value indicating better health. Directly, 3 months, 6 months and 9 months after the intervention the VAS score increased by 11.68 [95\% Cl 9.38; 13.97], 12.20 [95\% Cl 9.78; 14.61], 9.70 [95\% Cl 7.24; 12.17] and 6.11 [95\% Cl 3.57; 8.65], respectively. These increases reflected a clinically relevant change. (Table 2)

Figure 2 illustrates the age, sex and BMI adjusted course of self-reported health state based on EuroQol VAS scores and utility index score.

Table 2

Adjusted association of timepoint of measurement with EQ-5D utility index and VAS*

\begin{tabular}{|c|c|c|}
\hline & $\begin{array}{l}\text { EQ-5D utility index } \\
\mathrm{B}[95 \% \mathrm{Cl}]\end{array}$ & $\begin{array}{l}\text { EQ-VAS } \\
\text { B [95\% Cl] }\end{array}$ \\
\hline \multicolumn{3}{|c|}{ Timepoint (reference=directly before treatment) } \\
\hline Directly after spa-treatment & $0.09[0.07 ; 0.11]^{1}$ & $11.68[9.38 ; 13.97]^{1}$ \\
\hline 3 months after treatment & $0.08[0.06 ; 0.10]^{1}$ & $12.20[9.78 ; 14.61]^{1}$ \\
\hline 6 months after treatment & $0.06[0.05 ; 0.09]^{1}$ & $9.70[7.24 ; 12.17]^{1}$ \\
\hline 9 months after treatment & $0.04[0.02 ; 0.06]^{1}$ & $6.11[3.57 ; 8.65]^{1}$ \\
\hline Age, mean (SD); range & $-0.001[-0.001 ; 0.000]$ & $-0.18[-0.26 ;-0.11]^{1}$ \\
\hline Sex (reference=women) & $0.01[-0.00 ; 0.03]$ & $2.21[0.56 ; 3.87]^{1}$ \\
\hline Body Mass Index & $-0.002[-0.004 ;-0.001]^{1}$ & $-0.22[-0.43 ;-0.04]^{1}$ \\
\hline \multicolumn{3}{|c|}{ 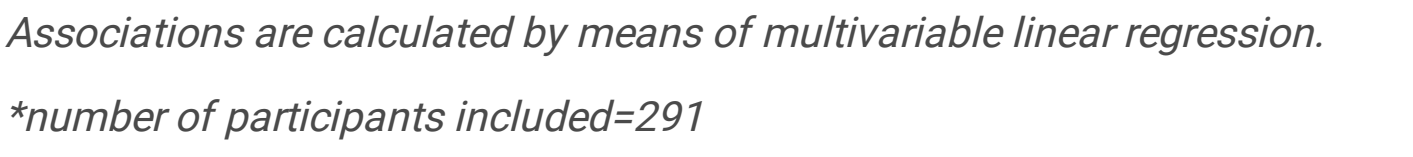 } \\
\hline \multicolumn{3}{|l|}{${ }^{1}$ significant at $p \leq 0.05$} \\
\hline \multicolumn{3}{|c|}{ Clinically relevant improvement (i.e. $\geq 0.05$ ) is indicated using bold font. } \\
\hline
\end{tabular}

\section{Discussion}


To our knowledge, this is the first time that systematically collected registry data have been used to explore the association between spa-therapy including radon with alterations in QoL in AS patients over a period of 9 months. Significant improvements in QoL were seen immediately and were sustained until 9 months after the intervention. These improvements were clinically relevant until 6 months after the intervention in case of the EQ-5D utility index and until 9 months after the intervention in case of the EQVAS. Generally, these findings are in agreement with other studies focussing on the benefits of spa therapy for QoL in AS patients. $(13,14,22,23)$.

Some differences were found in the course of the utility index compared to the course of the VAS-score. The utility index showed the largest improvement directly after the intervention while the VAS score was highest 3 months after the intervention. The latter is in agreement with other publications focussing on symptom relief and alterations in QoL through spa-therapy including radon which show a delayed therapy response. For example, van Tubergen et al. found the same delay when focussing on the EQ-5D utility index (14). However, in our study the delay was only observed in the VAS-score. A possible explanation might be found in the different population preferences accounted for in the calculation of utilities in the current study (German preference weights) compared to the study of van Tubergen et al. (Dutch preference weights). Yet, the unadjusted illustration of the 5 dimensions of health prior to the application of preference weights shows the same course of improvement suggesting that another explanation is more likely. Selection bias might be one: The observation may be attributable to the specific population included. Provision of data for the radon registry is voluntarily and participants included in the current study had provided complete data at all timepoints. This might indicate high motivation attributable to favourable treatment effects that are more precisely represented by the utility index than by the VAS score. An interesting side finding was that men had significantly higher EQ-VAS scores compared to women independent of their age, BMI or the timepoint of measurement. Previous research showed, that women with AS have less improvement in AS related outcome measures compared to men. However, the reason for this phenomenon remained unclear (31). In the current study the difference between men and women was not clinically relevant and only occurred in case of the VAS but not the utility index, which might suggest that perception of health plays a relevant role.

Clinically relevant changes attributable to spa therapy including radon have, to our knowledge, not been addressed by previous studies. The current evidence points out that in our cohort of AS-patients clinically relevant improvements in QoL can be sustained until 6 months (utility index) or even 9 months (VAS) after intervention. From a clinical perspective, this indicates the benefits of a repetitive treatment pattern. To achieve stable results, a periodic intervention should be scheduled every 6 months.

\section{Limitations and Strengths}

As in all studies based upon registry data limitations arise from the fact that data collection is not monitored or performed by the researcher and that data on confounders is somewhat limited. (32)

In the current study data on the frequency of interventions prior to the first timepoint of measurement were not systematically collected. This might have resulted in biased baseline values as participants who 
have received the intervention repeatedly likely have a better baseline health state than those who receive the intervention for the first time leading to a potential underestimation of the improvement in first-time participants. Confounders in the association of QoL with AS have been identified in previous literature and might have affected the current analyses as well. For example, a lower level of education and being a smoker is associated with lower QoL but this information was not available.(33)

Strengths of the study include a relatively large study sample with complete data over an extended period of time as well as the independence of data collection. Since data on the effectiveness of spa therapy including radon on the improvement of QoL in AS patients is still scarce, the current study provides relevant insights and opportunities for further research among other patient populations, and in comparison with usual care.

\section{Conclusion}

In conclusion, the current study reveals that spa-therapy including low-dose radon is beneficial in improving QoL in patients with AS and that these improvements are sustained for up to 9 months. It may be considered a valuable (complementary) treatment option for this purpose. Extrapolation of the results may support the decision of policy makers and insurances to refund bi-annual spa therapy including radon for patients with AS.

\section{Declarations}

\section{Ethics approval and consent to participate}

The study was approved by the Ethics Committee of the County of Salzburg (No 415-E/1966/32015). All participants provided informed consent prior to their participation. The conduct of the study, the acquisition, provision and analyses of data in this study are in agreement with the European General Data Protection Regulation 2018 and in accordance with the Declaration of Helsinki.

\section{Consent for publication}

N/A

\section{Availability of data and materials}

The datasets used and/or analysed during the current study are publicly available from the open data storage platform Zenodo using the following link: https://doi.org/10.5281/zenodo.5926209.

\section{Competing interest}

The authors have no conflicts of interest to disclose.

\section{Funding}


This research did not receive any specific grant from funding agencies in the public, commercial, or notfor-profit sectors.

\section{Author contribution statement}

AvZ conceptualized the current study, conducted and interpreted the analyses, wrote the initial draft of the manuscript and finalized its current version.

VS; HD and JF prepared and organized the data before analysis, were involved in the conceptual framework and protocol for the original data collection and reviewed and critically commented on the manucript. JU; WF; MK; SE and MO were involved in the recruitment of participants, reviewed and critically commented on the manuscript. MR, BH and MG were involved in the concept of the current study and the design for the original data collection, reviewed and critically commented on the manuscript. All authors agreed on the final version before submission.

\section{Abbreviations}

AS, Ankylosing spondylitis; BMI, Body Mass Index; Cl, confidence interval; EQ, EuroQol; QoL, Quality of life; RnIR, Radon indication registry for the assessment of pain reduction, increase of quality of life and improvement in body functionality throughout low-dose radon hyperthermia therapy; VAS, Visual Analogue Scale

\section{Acknowledgements}

We want to thank various people for their contribution to this study, especially Margret Schmid, Heike Thies, Roswitha Mayer, Christine Söllner and Claudia Pacher for organisational work. We are grateful for the assistance in data collection by Celina Ablinger, Julia Preishuber-Pflügl, Florentina Egger and Marlena Beyreis. We thank all physicians and medical technical assistants of Stiftung Kurtherme Badehospiz, Healing Gallery Bad Gastein, Cure and Rehabilitation Center Bad Hofgastein and Baerenhof Health Care \& Rehabilitation Center for their assistance during this project.

\section{References}

1. Rusman T, van Vollenhoven RF, van der Horst-Bruinsma IE. Gender Differences in Axial Spondyloarthritis: Women Are Not So Lucky. Curr Rheumatol Rep. 2018;20(6):35.

2. Dean LE, Jones GT, MacDonald AG, Downham C, Sturrock RD, Macfarlane GJ. Global prevalence of ankylosing spondylitis. Rheumatology (Oxford). 2014;53(4):650-7.

3. Braun J, Sieper J. Ankylosing spondylitis. Lancet. 2007;369(9570):1379-90.

4. van Genderen S, Plasqui G, van der Heijde D, van Gaalen F, Heuft L, Luime J, et al. Social Role Participation and Satisfaction With Life: A Study Among Patients With Ankylosing Spondylitis and Population Controls. Arthritis Care Res (Hoboken). 2018;70(4):600-7. 
5. Calvert MJ, Freemantle N. Use of health-related quality of life in prescribing research. Part 1: why evaluate health-related quality of life? J Clin Pharm Ther. 2003;28(6):513-21.

6. Addington-Hall J, Kalra L. Who should measure quality of life? BMJ. 2001;322(7299):1417-20.

7. Tanski W, Swiatoniowska-Lonc N, Dudek K, Jankowska-Polanska B. Benefit of Biological Drugs for Quality of Life in Patients with Ankylosing Spondylitis: A Systematic Review and Meta-Analysis of Clinical Trials. Adv Exp Med Biol. 2021;1335:63-78.

8. Kotsis K, Voulgari PV, Drosos AA, Carvalho AF, Hyphantis T. Health-related quality of life in patients with ankylosing spondylitis: a comprehensive review. Expert Rev Pharmacoecon Outcomes Res. 2014;14(6):857-72.

9. Gravaldi LP, Bonetti F, Lezzerini S, De Maio F. Effectiveness of Physiotherapy in Patients with Ankylosing Spondylitis: A Systematic Review and Meta-Analysis. Healthcare. 2022;10(1):132.

10. Packham J. Optimizing outcomes for ankylosing spondylitis and axial spondyloarthritis patients: a holistic approach to care. Rheumatology (Oxford). 2018;57(suppl_6):vi29-vi34.

11. Moder A, Foisner W, Hitzl W, Fagerer N, Ritter M, Kullich W. Schmerz, Krankenstände, Befindlichkeit, Medikamentenverbrauch und Funktionsverbesserung im Jahr vor und nach einer kombinierten Radonthermalkur. Physikalische Medizin, Rehabilitationsmedizin, Kurortmedizin. 2011;21(05):215-9.

12. Kamioka H, Nobuoka S, liyama J. Overview of Systematic Reviews with Meta-Analysis Based on Randomized Controlled Trials of Balneotherapy and Spa Therapy from 2000 to 2019. Int J Gen Med. 2020;13:429-42.

13. Colina M, Ciancio G, Garavini R, Conti M, Trotta F, Govoni M. Combination treatment with etanercept and an intensive spa rehabilitation program in active ankylosing spondylitis. Int $\mathrm{J}$ Immunopathol Pharmacol. 2009;22(4):1125-9.

14. Van Tubergen A, Boonen A, Landewe R, Rutten-Van Molken M, Van Der Heijde D, Hidding A, et al. Cost effectiveness of combined spa-exercise therapy in ankylosing spondylitis: a randomized controlled trial. Arthritis Rheum. 2002;47(5):459-67.

15. Lind-Albrecht G. Der therapeutische Einsatz von Radon. arthritis +rheuma. 2007;27:272-8.

16. Lind-Albrecht G. Einfluss der Radonstollentherapie auf Schmerzen und Verlauf bei Spondylitis ankylosans (M. Bechterew): eine randomisierte prospektive Studie1994.

17. Ruhle PF, Wunderlich R, Deloch L, Fournier C, Maier A, Klein G, et al. Modulation of the peripheral immune system after low-dose radon spa therapy: Detailed longitudinal immune monitoring of patients within the RAD-ON01 study. Autoimmunity. 2017;50(2):133-40.

18. Ruhle PF, Klein G, Rung T, Tiep Phan H, Fournier C, Fietkau R, et al. Impact of radon and combinatory radon/carbon dioxide spa on pain and hypertension: Results from the explorative RAD-ON01 study. Mod Rheumatol. 2019;29(1):165-72.

19. Kullmann M, Ruhle PF, Harrer A, Donaubauer A, Becker I, Sieber R, et al. Temporarily increased TGFbeta following radon spa correlates with reduced pain while serum IL-18 is a general predictive marker for pain sensitivity. Radiat Environ Biophys. 2019;58(1):129-35. 
20. Moder A, Hufnagl C, Lind-Albrecht G, Hitzl W, Hartl A, Jakab M, et al. Effect of combined Low-Dose Radon- and Hyperthermia Treatment (LDRnHT) of patients with ankylosing spondylitis on serum levels of cytokines and bone metabolism markers: a pilot study. Int J Low Radiat. 2010;7(6):423-35.

21. Lange U, Dischereit G, Tarner I, Frommer K, Neumann E, Muller-Ladner U, et al. The impact of serial radon and hyperthermia exposure in a therapeutic adit on pivotal cytokines of bone metabolism in rheumatoid arthritis and osteoarthritis. Clin Rheumatol. 2016;35(11):2783-8.

22. Falkenbach A, Kovacs J, Franke A, Jorgens K, Ammer K. Radon therapy for the treatment of rheumatic diseases--review and meta-analysis of controlled clinical trials. Rheumatol Int. 2005;25(3):205-10.

23. Franke A, Franke T. Long-term benefits of radon spa therapy in rheumatic diseases: results of the randomised, multi-centre IMuRa trial. Rheumatol Int. 2013;33(11):2839-50.

24. Rabin R, de Charro F. EQ-5D: a measure of health status from the EuroQol Group. Ann Med. 2001;33(5):337-43.

25. Ludwig K, Graf von der Schulenburg JM, Greiner W. German Value Set for the EQ-5D-5L. Pharmacoeconomics. 2018;36(6):663-74.

26. Laxy M, Teuner $\mathrm{C}$, Holle R, Kurz $\mathrm{C}$. The association between BMI and health-related quality of life in the US population: sex, age and ethnicity matters. Int J Obes (Lond). 2018;42(3):318-26.

27. Gordeev VS, Maksymowych WP, Evers SM, Ament A, Schachna L, Boonen A. Role of contextual factors in health-related quality of life in ankylosing spondylitis. Ann Rheum Dis. 2010;69(1):108-12.

28. Levinsky M, Schiff M. Lifetime cumulative adversity and physical health deterioration in old age: Evidence from a fourteen-year longitudinal study. Soc Sci Med. 2021;289:114407.

29. Dolan P. Modeling valuations for EuroQol health states. Med Care. 1997;35(11):1095-108.

30. Luo N, Chew LH, Fong KY, Koh DR, Ng SC, Yoon KH, et al. Do English and Chinese EQ-5D versions demonstrate measurement equivalence? An exploratory study. Health Qual Life Outcomes. 2003;1:7.

31. van der Horst-Bruinsma IE, Zack DJ, Szumski A, Koenig AS. Female patients with ankylosing spondylitis: analysis of the impact of gender across treatment studies. Ann Rheum Dis. 2013;72(7):1221-4.

32. Thygesen LC, Ersbøll AK. When the entire population is the sample: strengths and limitations in register-based epidemiology. European journal of epidemiology. 2014;29(8):551-8.

33. Bodur H, Ataman S, Rezvani A, Bugdayci DS, Cevik R, Birtane M, et al. Quality of life and related variables in patients with ankylosing spondylitis. Qual Life Res. 2011;20(4):543-9.

\section{Figures}




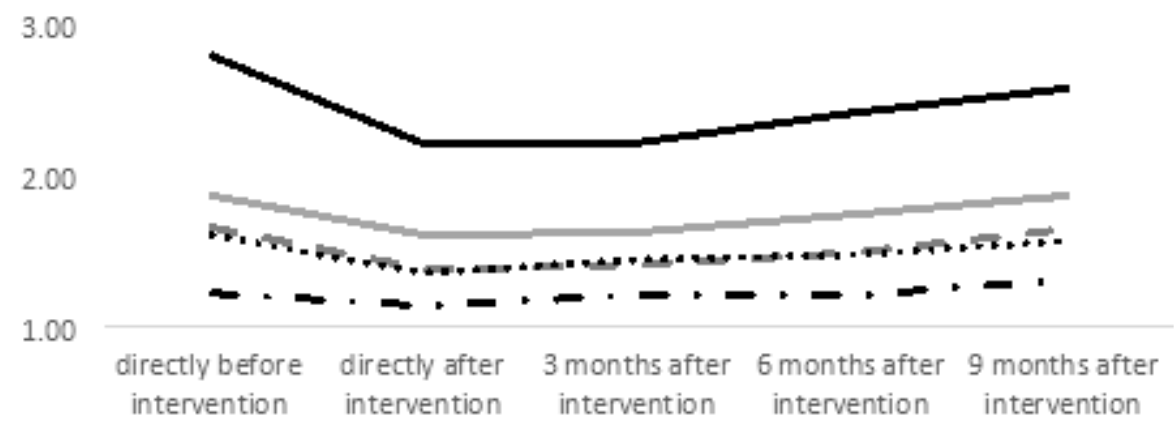

\section{Figure 1}

Unadjusted average course of health based on EQ-5D health dimensions (dimension score range 1-5 with lower scores representing better health)

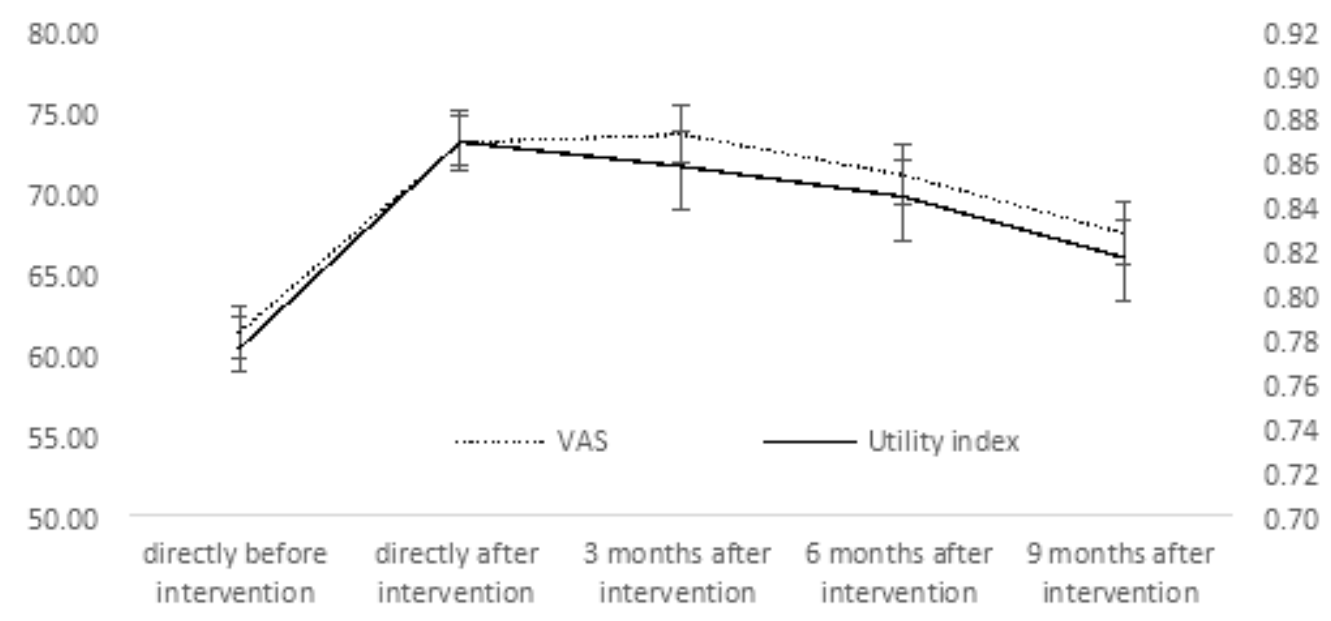

\section{Figure 2}

Age, sex and BMI adjusted course of self-reported health state based on EuroQol VAS scores and utility index score (utility index range 0-1; VAS-score range 0-100 with higher scores representing better health) 